\title{
Forest Fire Detection through Wireless Sensor Network using Type-2 Fuzzy System
}

\author{
A.K. Singh \\ Department of Electronics Engineering \\ IIIT Allahabad, India
}

\author{
Harshit Singh \\ Department of Information Technology \\ IIIT Allahabad, India
}

\begin{abstract}
Fire detection is always been a crucial challenge for human, moreover detecting fire using automated sensors definitely requires efficient and accurate ways. Since fire depends on more than one physical/environmental condition simultaneously, so in this paper we have used fuzzy type-2 logic for fire detection. Fuzzy gives best results in such cases because there is an uncertainty about how much extent of a factor like temperature, humidity and light intensity should be involved to cause a fire. In addition to this interval type-2 fuzzy system is used to make the results accurate and error free so that there would be no uncertainty in decision making.
\end{abstract}

Keywords- fire detection, fuzzy type-2 system, wireless sensor network.

\section{INTRODUCTION}

Wireless sensor network (WSN) consists of sensor nodes having the abilities of sensing, communication and processing. These sensor nodes are deployed and distributed randomly over the area which has to be observed. These nodes are automated, having limited energy and memory. Sensor nodes can sense multiple physical, environmental conditions like temperature, motion, pressure, humidity etc and pass the information to the base station. As the resources are limited, we should use it wisely and efficiently. A huge amount of work has been done on lifetime enhancement of sensor nodes and efficient use of WSN. Wireless sensor network is being used in various fields. Due to advancement in technology and efficient ways of using WSN, it is being used in numerous applications like security, wild-life protection, environment monitoring, motion detection, radioactivity and various other military and industrial applications. For efficient use of WSN all nodes are divided into small groups known as clusters, each cluster have a cluster head which communicates with the base station, in this way there would be no need to send data from each sensor node to base station. Nodes will send their sensed data to their respective cluster heads and hence lots of energy could be utilized in further calculations. All the sensor nodes in the network work co-operatively. Keeping the fact in into mind that sending information from node to the base station would consume more energy, so simple computations and data fusion is done by the nodes itself and the information is sent to their respective cluster heads, which are responsible for sending data to the base station.

In this paper we have used fuzzy logic for detecting fire. Fuzzy gives best results in such systems which do not have exact mathematical model. Fuzzy takes less memory, less processing and less energy. The reasoning in fuzzy logic is very similar to human reasoning. Modeling of fuzzy system is done in natural language, which makes fuzzy logic easy to understand and better to implement.

Type-1 fuzzy logic controllers have been applied to many different applications successfully till date. However there is a need to cope with large amount of uncertainties. Type-2 fuzzy system can handle such uncertainties and gives a better and accurate result. Fuzzy system comprises of mainly three parts namely fuzzufication, inference rules and defuzzification. Converting a crisp value to a fuzzy value is called Fuzzification. In this process the crisp numbers is given as input and fuzzified accordingly by using given membership function. Membership functions can be triangular, trapezoidal or Gaussian as required.

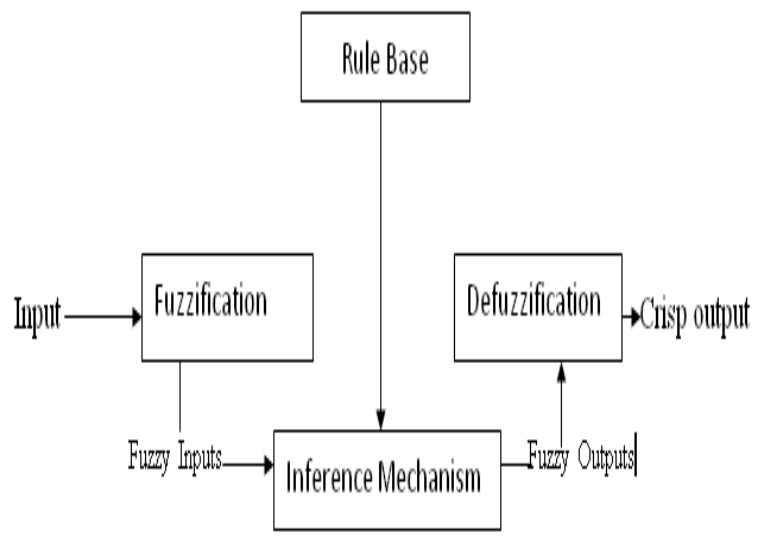

Figure 1. Block diagram of type-1 fuzzy system 
Inference rules are the heart of every fuzzy system, after the input gets fuzzified mapping of fuzzified input to an output according to IF-THEN rules is done according to rule base table. The IF part is known as antecedent and THEN part is known as consequent. After applying rules defuzzifier gives a crisp output from the fuzzy set that is the output of inference engine.

Following figure shows block diagram for type-2 fuzzy system. There is slight difference in type- 1 and type- 2 fuzzy system. In type-2, the sets we get after fuzzification are type-2 fuzzy sets, after applying inference rules on those sets the output is again type- 2 fuzzy sets, therefore before defuzzification, type reduction is done. The output after type reduction is type-1 fuzzy set, which can be defuzzified into crisp output.

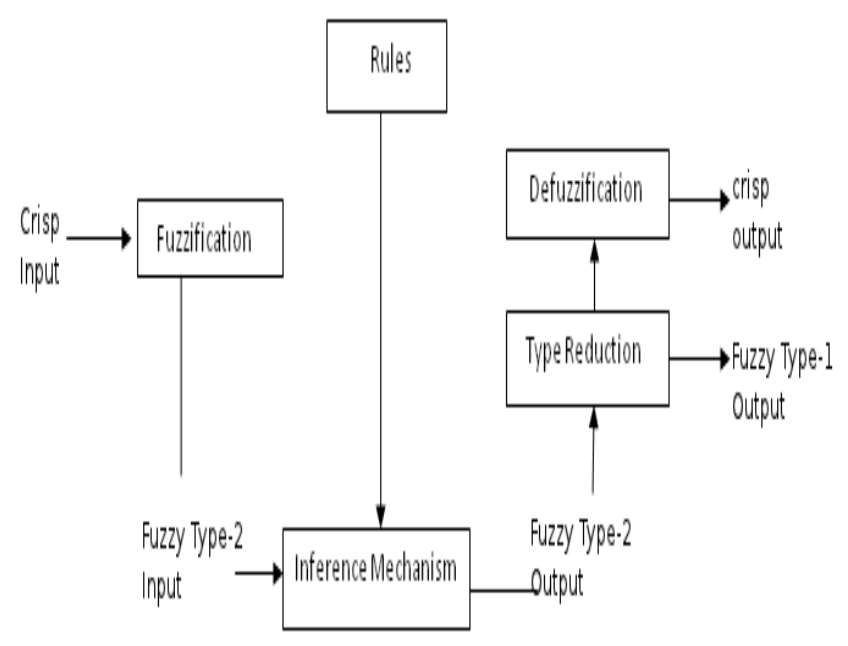

Figure 2. Block diagram of type-2 fuzzy system

The concept of type-2 fuzzy system can be understood from the figure below.

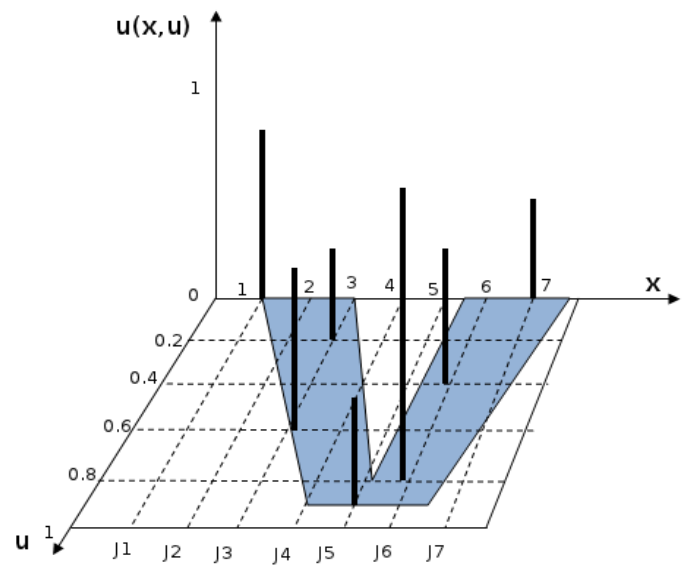

Figure 3. Representation of type-2 fuzzy logic.
For each $\mathrm{x}$ there is a primary membership grade in $\mathrm{u}$ axis and each $u$ is having a secondary membership grade in $\mu_{\mathrm{A}}(\mathrm{x}, \mathrm{u})$. Type- 2 provides additional degree of freedom for modeling uncertainties. There are further two types of type-2 fuzzy system, 1) general type-2 system, 2) interval type-2 system. A general type-2 system is described in figure2. In interval type-2 fuzzy system we assume that the secondary membership grade i.e. $\mu_{\mathrm{A}}(\mathrm{x}, \mathrm{u})$ value is same for all $\mathrm{J}$. Which means the third dimension does not have any important information. The shaded part in the above figure is used in interval type-2 fuzzy system for calculation. Unlike type-1 fuzzy system, type-2 fuzzy logic is more complicated to understand and implement. But it can handle and model more uncertainties than type-1 fuzzy system. Fuzzy type-2 provides better and accurate results as compared to type-1. In type- 2 fuzzy logic the membership functions are also fuzzy.

The remaining of this paper is organized as follows. Related work is discussed in section II. Proposed work is explained in section III. Section IV discusses simulation results. Finally section $\mathrm{V}$ presents the conclusion.

\section{RELATED WORK}

Most of the research work in wireless sensor network is done towards enhancing and increasing the lifetime of sensor network by proposing new energy efficient ways. In [1] the authors have used fuzzy inference for fire detection in aircrafts. A fire detection technique using neural network and fuzzy inference is introduced in [2]. Type-1 fuzzy system is used to detect fire in WSN [3], in this paper author have used four parameters temperature, humidity, light intensity and carbon mono-oxide density to find out the probability of fire using type-1 fuzzy inference.

In 1975 Zadeh introduced type-2 fuzzy sets [4], after that type- 2 becomes a hot topic for researchers, a lot of research works have been done on comparison between the performances and results of type- 1 and type-2 fuzzy systems [5], it has been found that type-2 fuzzy gives better results when we have to consider large amount of uncertainty. Since type- 2 is hard to implement it is being implemented and used by few applications which needed accuracy in their results for decision making.

In [6] Mendel has presented very comprehensive survey about type-2 fuzzy sets and systems. It describes all the important issues regarding type-2 fuzzy sets and its calculation. Everything related to type-2 fuzzy like general and interval type-2 systems, membership grades (primary and secondary both), footprint of uncertainty (FOU) representation, inference rules, calculation of switch points, type reduction and defuzzification is described in a very understandable way. Dongrui $\mathrm{Wu}$ presented a new way of representing a type-2 fuzzy membership function by using 9 points. This approach of type-2 membership function representation is very 
helpful and quite simple to implement. Since energy efficiency is always being an important point to be considered by the researchers, so [7] describes the comparison between the proposed type reduction algorithms till date and introduced a better algorithm for the same.

\section{PROPOSED WORK}

In this paper interval type-2 fuzzy logic is implemented for fire detection. An interval type-2 fuzzy system can be represented in two dimensions. As described in [6] the figure of interval type- 2 membership function is given below.

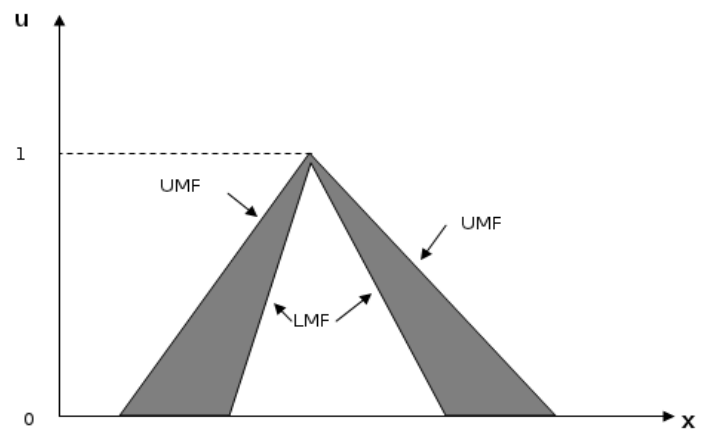

Figure 4. Representation of UMF, LMF and FOU

In Figure 3, Upper Membership Function (UMF) and Lower Membership Function (LMF) is shown. The area between UMF and LMF is known as Footprint Of Uncertainty (FOU).

In this paper we have used the approach described by Dongrui Wu for designing a type-2 fuzzy membership function with the help of nine points.

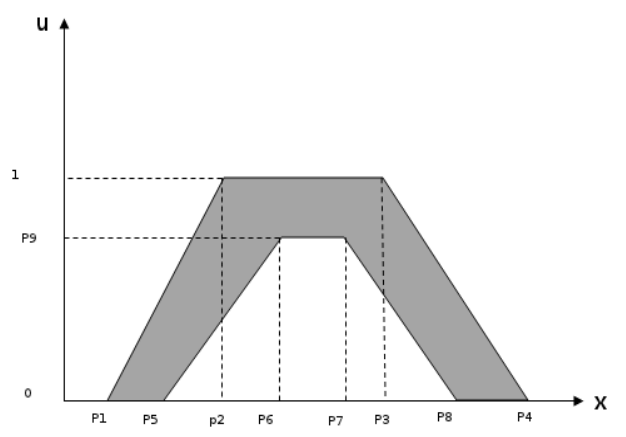

Figure 5. Representation of a type-2 membership function using nine points

For detecting fire we have taken four input factors in our account as in [3] i.e. temperature, light intensity, humidity and carbon mono-oxide density. Each input parameter is divided into three membership functions low, medium and high. While the output, which is probability of fire, is divided into five membership functions very low, low, medium, high and very high. The simulation is done by using MATLAB. Membership functions used for simulation are the combination of triangular membership functions and trapezoidal membership functions.

Fuzzification: The figures of input variables of type-2 fuzzy inference system are shown here.

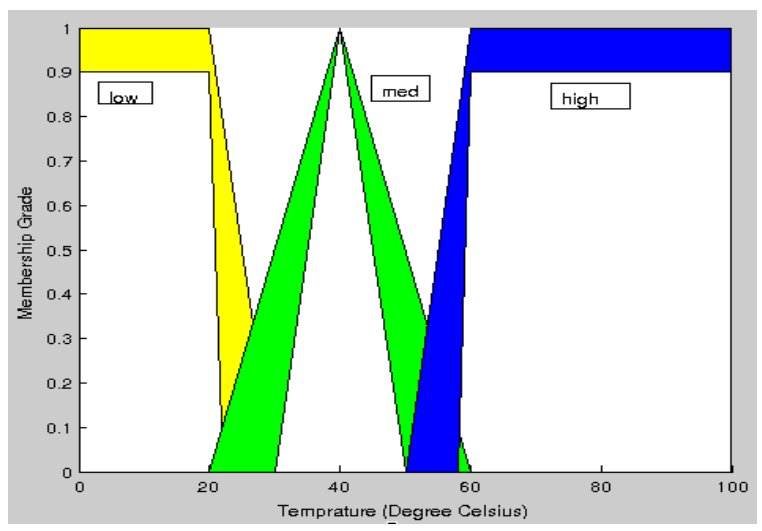

Figure 6. Membership functions for temperature

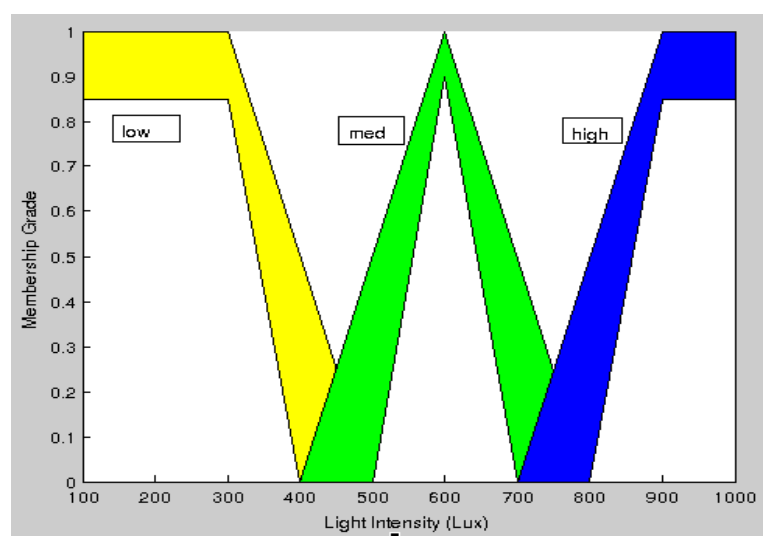

Figure 7. Membership Functions for Light Intensity

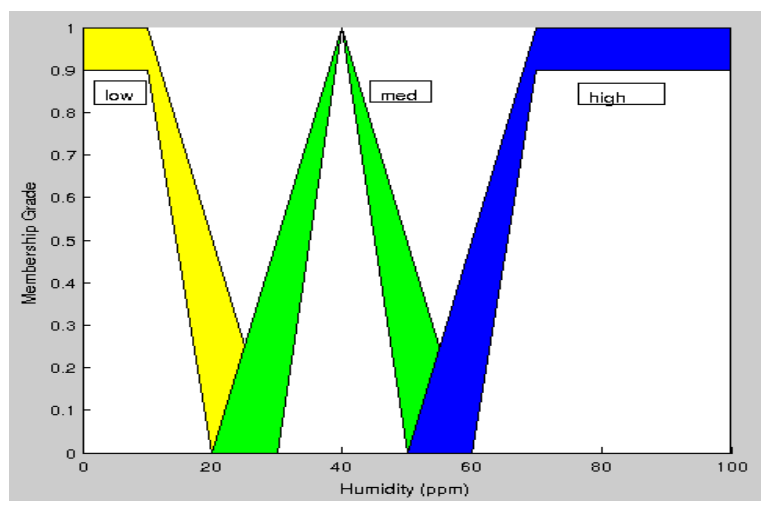

Figure 8. Membership Functions for Humidity 


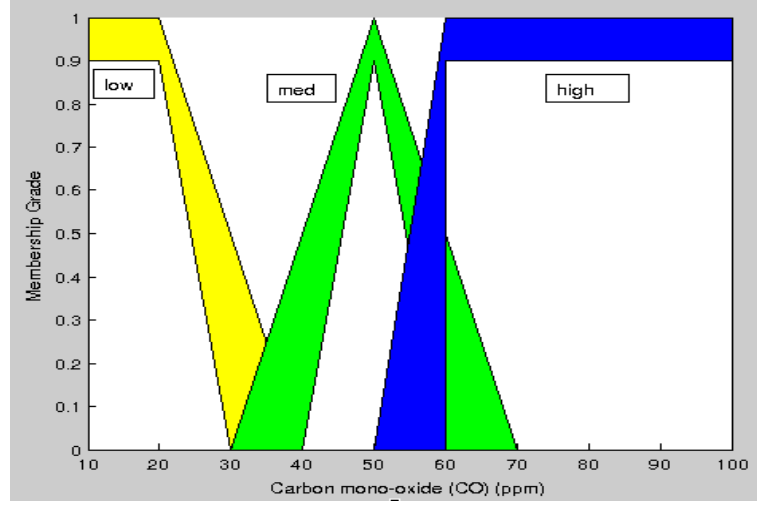

Figure 9. Membership Functions for CO density

The figure of output variable of type-2 fuzzy inference system is shown in Figure 9.

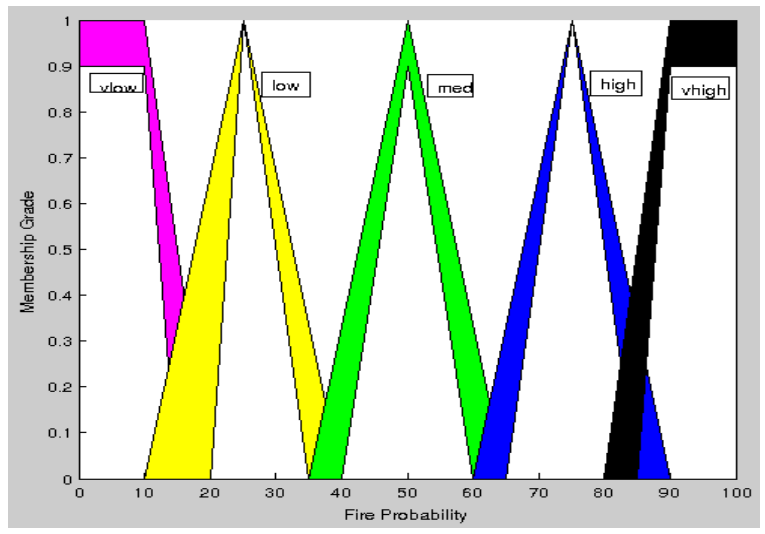

Figure 10. Membership functions for probability of fire

Inference Rules: As in type-1 fuzzy system, rules are in the form in IF-THEN form, in type-2 inference system also rules are in the same form just the difference is that in type-2 fuzzy system sets involved in antecedent and consequent parts are type- 2 fuzzy sets. For example, rule Rl: IF $x_{1}$ is $f_{1}^{1}$ and $x_{2}$ is $f_{2}^{1}$ and ....... $x_{k}$ is $f_{k}^{1}$, THEN $\mathrm{y}$ is $\mathrm{G}^{\mathrm{l}}$, where $\mathrm{x}_{\mathrm{i}} \mathrm{s}$ are crisp inputs; $\mathrm{f}_{\mathrm{i}}^{\mathrm{l}} \mathrm{s}$ are antecedent sets $(\mathrm{I}=1, \ldots, \mathrm{k}) ; \mathrm{y}$ is output and $\mathrm{G}^{\mathrm{l}} \mathrm{s}$ are consequent sets. The consequent sets are in the form of $\left[\mathrm{y}_{\mathrm{n}}, \mathrm{y}_{\mathrm{n}}\right]$, where $\mathrm{y}_{\mathrm{n}}$ and $\mathrm{y}_{\mathrm{n}}$ are centroids of lower membership function and upper membership function respectively of the consequent type-2 membership function as described in [8]. In many cases $\mathrm{y}_{\mathrm{n}}$ can be equal to $\mathrm{y}_{\mathrm{n}}$ i.e. consequent can be a crisp value. In the proposed model there are four input variables each having three membership functions so the number of rules is $3^{4}=81$. For example R1: IF temperature is low and light intensity is low and humidity is low and CO density is low, THEN P(fire) is very low. R2: IF temperature is high and light intensity is high and humidity is low and CO density is high, THEN P(fire) is very high. On the basis of these rules output set is obtained on which type reduction and defuzzification is applied to get a single crisp number as output.

Type Reduction and Defuzzification: Mostly KarnikMendel Algorithm is used for type reduction. In the last few years researchers have proposed many algorithms for efficient type reduction. In this paper we have simulated Enhanced Iterative Algorithm with Stop Condition (EIASC) [7]. From the consequent set [ $\mathrm{y}_{\mathrm{n}}$, $\left.\mathrm{y}_{\mathrm{n}}\right] \mathrm{yl}$ and $\mathrm{yr}$ are obtained and switch points L and R are determined by

$$
\begin{gathered}
\mathrm{y}^{\mathrm{L}}<=\mathrm{y}_{\mathrm{l}}<=\mathrm{y}^{\mathrm{L}+1} \\
\mathrm{y}^{\mathrm{R}}<=\mathrm{y}_{\mathrm{r}}<=\mathrm{y}^{\mathrm{R}+1}
\end{gathered}
$$

For defuzzification $\mathrm{y}_{1}$ and $\mathrm{y}_{\mathrm{r}}$ values are used. The crisp output is calculated by

$$
\mathrm{y}=\left(\mathrm{y}_{1}+\mathrm{y}_{\mathrm{r}}\right) / 2
$$

After the simulation of type-2 fuzzy system it is being observed that the result obtained i.e. the probability of fire is different for many inputs if compared with type-1 simulation results, which simply means that the simulation results of type- 2 fuzzy system are more accurate and efficient than type-1 fuzzy system.

\section{SIMULATION RESULTS}

The results are crisp number from the set $[0,100]$ which is the scale of fire probability. Decision making could be done on the basis of output obtained by making few conditions.

1. If the output is between 0 to 15 , then the probability of fire is very low.

2. If the output is between 16 to 35 , then the probability of fire is low.

3. If the output is between 36 to 60 , then the probability of fire is medium.

4. If the output is between 61 to 85 , then the probability of fire is high.

5. If the output is between 86 to 100 , then the probability of fire is very high.

For example if the input is [70 86513 81], where first, second, third and forth element of input matrix represents temperature, light intensity, humidity and carbon mono-oxide density respectively, the output is 93.6 and probability of fire is very high. If the input is [32 698 18 7] output is 50.5 and probability of fire is medium.

Figures. 11 to 13 shows a control surface of a fire probability based on the input parameters 


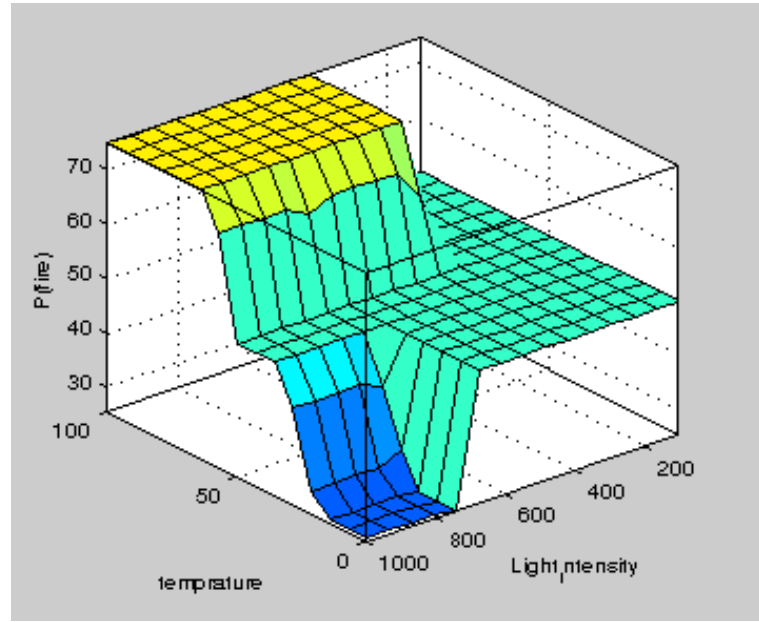

Figure 11

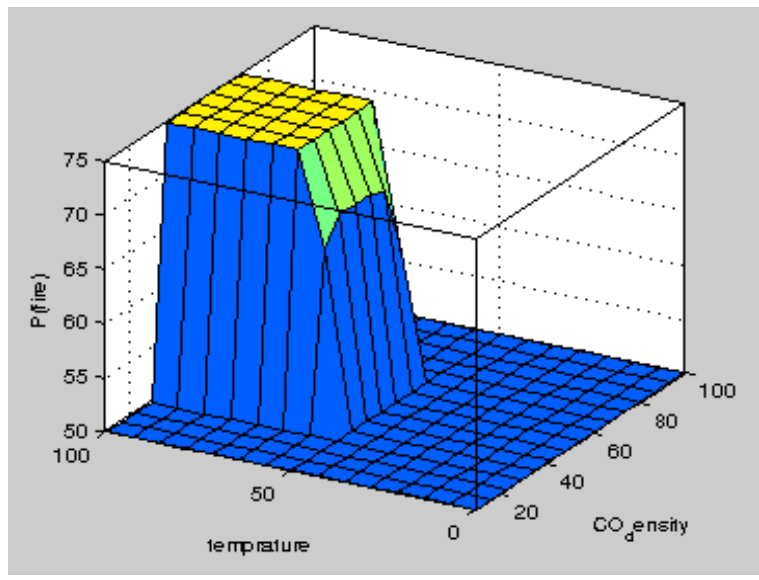

Figure 12

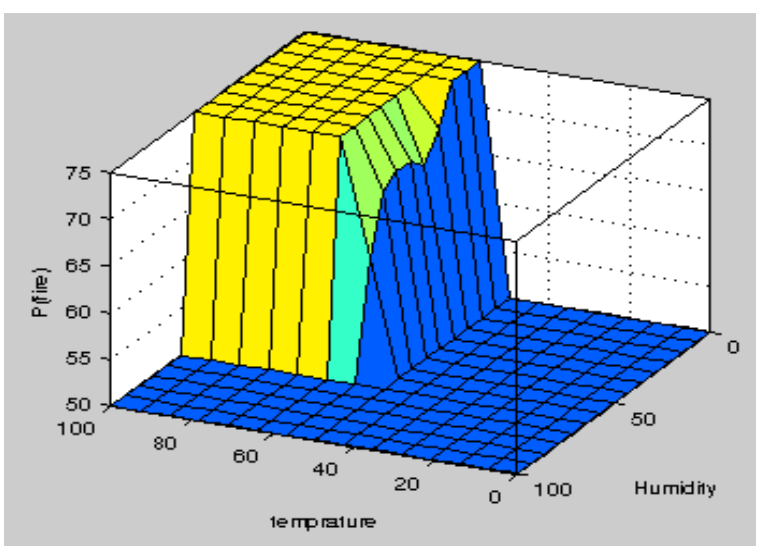

Figure 13

\section{CONCLUSION}

In this paper, we proposed a fuzzy type-2 approach for fire detection using wireless sensor network. Our proposed fuzzy type-2 logic approach handles the uncertainty present in the data effectively and gives the best results with very low false alarm rate. The decision based on this approach is more accurate. Furthermore, the results obtained are more accurate than the results obtained from type-1 fuzzy system. The membership functions and the parameters can be changed and modified as required. Rules also could be altered and adjusted according to parameters for further work on this model.

\section{REFERENCES}

[1] Y. Simon, "A fuzzy logic approach to fire detection in aircraft dry bays and engine compartments," IEEE Transaction on Industrial Electronics, vol. 47, no. 5, pp. 1161-1171, Oct 2000

[2] Z. B. Li and H. Zhou, "Research on the application of fuzzy data fusion to cable fire detecting system," in Proceedings of the Third International Conference on Machine Learning and Cybernetics, vol. 4, Shanghai, Aug 2004, pp. 2083- 2085

[3] P. Manjunatha, A.K. Verma and A. Srividya, "Multi-Sensor Data Fusion in Cluster based Wireless Sensor Networks Using Fuzzy Logic Method," 2008 IEEE Region 10 Colloquium and the Third ICIIS, Kharagpur, INDIA December 810.

[4] L.A. Zadeh, "The concept of a linguistic variable and its application to approximate reasoning- 1," Information Sciences, vol. 8, pp. 199-249, 1975.

[5] J.M.Mendel, "A quantitative comparison of interval type-2 and type-1 fuzzy logic systems: First results," 2010 IEEE International Conference on Fuzzy Systems, pp. 1-8, July 2010.

[6] Jerry M. Mendel, "Type-2 fuzzy sets and systems: An overview," IEEE computational intelligence magazine, vol.2, no. 1, pp. 20-29, February 2007.

[7] Dongrui U, Maowen Nie "Comparison and Practical Implementation of Type-Reduction Algorithms for Type-2 Fuzzy Sets and Systems" 2011 IEEE International Conference on Fuzzy Systems June 27-30, 2011, Taipei, Taiwan

[8] N. N. Karnik and J. M. Mendel, "Centroid of a type-2 fuzzy set," Information Sciences, vol. 132, pp. 195-220, 2001. 